\title{
Euclidean Calculation of Feature Points of a Rotating Satellite: A Daisy-Chaining Approach
}

\author{
Keith Dupree,,- Nicholas R. Gans, \pm William MacKunis, \pm and Warren E. Dixon $\underline{\S}$ \\ University of Florida, Gainesville, Florida 32622-6250
}

\begin{abstract}
DOI: $\underline{10.2514 / 1.33087}$
The occlusion of feature points and/or feature points leaving the field of view of a camera is a significant practical problem that can lead to degraded performance or instability of visual servo control and vision-based estimation algorithms. By assuming that one known Euclidean distance between two feature points in an initial view is available, homography relationships and image geometry are used in this paper to determine the Euclidean coordinates of feature points in the field of view. A new daisy-chaining method is then used to relate the position and orientation of a plane defined by the feature points to other feature-point planes that are rigidly connected. Through these relationships, the Euclidean coordinates of the original feature points can be tracked even as they leave the field of view. This objective is motivated by the desire to track the Euclidean coordinates of feature points on one face of a satellite as it continually rotates and feature points become self-occluded. A numerical simulation is included to demonstrate that the Euclidean coordinates can be tracked even when they leave the field of view. However, the results indicate the need for a method to reconcile any accumulated error when the feature points return to the field of view.
\end{abstract}

\section{Nomenclature}

\begin{tabular}{|c|c|c|}
\hline & 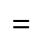 & ion matrix \\
\hline$d_{j}^{*}$ & $=$ & distance to $\pi_{j}^{*}$ plane along $n_{j}^{*}$ \\
\hline $\mathcal{F}_{j}, \mathcal{F}_{j}^{*}$ & $=$ & d to the $\pi_{j}$ and $\pi_{j}^{*}$ planes \\
\hline & $=$ & projective homography matrix of the $j$ th frame \\
\hline$H_{j}$ & $=$ & Euclidean homography matrix of the $j$ th frame \\
\hline & $=$ & fixed coordinate frame attached to the camera \\
\hline$m_{j i}, m_{j i}^{*}$ & $=$ & $\begin{array}{l}\text { he } i \text { th feature } \\
\text { ed in } \mathcal{I}\end{array}$ \\
\hline $\bar{m}_{j i}, \bar{m}_{j i}^{*}$ & $=$ & $\begin{array}{l}\text { Euclidean coordinate of the } i \text { th feature point of the } \\
\pi_{j} \text { and } \pi_{j}^{*} \text { planes expressed in } \mathcal{I}\end{array}$ \\
\hline$n_{j}^{*}$ & $=$ & unit normal vector to the $\pi_{j}^{*}$ plane expressed in $\mathcal{I}$ \\
\hline$p_{j i}, p_{j i}^{*}$ & & $\begin{array}{l}\text { coordinates for the } i \text { th feature } \\
\text { d } \pi_{j}^{*} \text { planes }\end{array}$ \\
\hline$R_{j}, R_{j}^{*}$ & $=$ & rotation from the $\pi_{j}$ and $\pi_{j}^{*}$ planes to $\mathcal{I}$ \\
\hline$R_{\mathcal{F} i}^{\mathcal{F} j}$ & $=$ & rotation from frame $i$ to frame $j$ \\
\hline $\mathrm{SO}($ & $=$ & by 3 orthogonal matrices. \\
\hline$s_{j i}$ & $=$ & $\begin{array}{l}\text { coordinates of the } i \text { th feature point of the } \pi_{j} \text { plane } \\
\text { expressed in } \mathcal{F}_{j}^{*}\end{array}$ \\
\hline & $=$ & vector to the origin of $\mathcal{F}_{j}$ and $\mathcal{F}_{j}^{*}$ expressed in $\mathcal{I}$ \\
\hline & & $\begin{array}{l}\text { ector from the origin of frame } i \text { to the origin of } \\
\text { ame } j \text {, expressed in frame } k\end{array}$ \\
\hline${ }_{j i}$ & $=$ & epth ratio of the $i$ th feature point of the $\pi_{j}$ plane \\
\hline & $=$ & \\
\hline & $=$ & when it first comes into the field of vie \\
\hline
\end{tabular}

Received 26 June 2007; revision received 2 January 2008; accepted for publication 2 January 2008. Copyright (C) 2008 by the American Institute of Aeronautics and Astronautics, Inc. All rights reserved. Copies of this paper may be made for personal or internal use, on condition that the copier pay the $\$ 10.00$ per-copy fee to the Copyright Clearance Center, Inc., 222 Rosewood Drive, Danvers, MA 01923; include the code 0731-5090/08 \$10.00 in correspondence with the CCC.

${ }^{*}$ Graduate Student, Department of Mechanical and Aerospace Engineering; kdupree@ufl.edu. Student Member AIAA.

Postdoctoral Researcher, Department of Mechanical and Aerospace Engineering; ngans@ufl.edu. Member AIAA.

${ }^{\ddagger}$ Graduate Student, Department of Mechanical and Aerospace Engineering; mackunis@ufl.edu. Student Member AIAA.

${ }^{\S}$ Assistant Professor, Department of Mechanical and Aerospace Engineering; wdixon@ufl.edu. Member AIAA.

\section{Introduction}

V ISION-BASED technologies commonly exploit image processing methods to identify feature points (e.g., corners or centroids) on a target of interest. These feature points are then used to determine the motion of the camera or target for image-based estimation and control methods. If the feature points leave the field of view (FOV) or become occluded, then degraded performance or instability of visual servo control and vision-based estimation algorithms can occur. The fundamental challenge and potential pervasive impact of developing methods to keep the feature points in the FOV has inspired a variety of research efforts. For example, the results in [1,2] exploit partitioned or switching visual servoing methods to keep the object in the FOV. In [3-8], potential fields or navigation functions are used to ensure the visibility of the feature points during closed-loop control. Zoom control is used in [9] to adjust the focal length of the camera to keep all features in the FOV during the control task. Some researchers have also investigated methods to enlarge the FOV. For example, image mosaicing is used in [10-13] to capture multiple images that are stitched together to obtain a larger image, and in [14], multiple images are fused from multiple cameras.

In addition to research focused on keeping the feature points in the FOV, other research has focused on methods that are invariant to occlusion or feature points leaving the FOV. For example, a linear probabilistic recursive estimation method is described in [15] for reconstructing three-dimensional structure and motion from an integrated sequences of images. In [16], a smooth task function with weighted features that allows visibility changes in the image features during the control task is developed. A variety of feature-pointtracking algorithms have also been proposed that can predict featurepoint locations (at least for short-time partial occlusions) based on shape information such as a CAD model and/or the time history of the object (i.e., the object dynamics). Some examples of these approaches are provided in [17-22].

An image-based estimation strategy is developed in this paper that uses the preliminary efforts in [23] to determine the Euclidean feature-point coordinates of features on a satellite by using multiple images from a single camera based on knowledge of one geometric length between two feature points. The motivation for this research arises from the need for image-based state estimation and tracking of a satellite, for which multiple feature points may become selfoccluded or leave the FOV; however, the development is generic to any rigid body. Image geometry and homography relationships are combined with a new daisy-chaining method $[24,25]$ to achieve the 
results. Specifically, image geometry and homography relationships are used to determine the Euclidean coordinates of nonoccluded feature points in the camera FOV, and the daisy-chaining method is used to link the successive images of the rigid body as feature points (i.e., different surfaces of the spinning satellite) come into and leave the FOV. A numeric simulation is included to demonstrate the developed algorithm.

The contribution of the developed method is the ability to estimate the Euclidean coordinates of the feature points even if the feature points (permanently) leave the FOV or become occluded. The developed estimation method is in contrast to previously described visual servo control methods that require camera actuation by switching, potential fields or navigation functions, or focal length adjustment/zoom control to force the feature points to remain in the FOV. Hence, the developed estimation method could be used to augment those methods. Another contribution of this work is that limited geometric knowledge of the object is required, in contrast to methods that exploit a CAD model or a dynamic model of the object.

\section{Geometric Model}

Consider a single camera observing the motion of a satellite that is initially identified by four coplanar and noncollinear feature points. Image processing techniques can often be used to select coplanar and noncollinear feature points within an image. However, if four coplanar target points are not available, then the subsequent development can also exploit the virtual parallax method [26], in which the noncoplanar points are projected onto a virtual plane.

To facilitate the ability to track the motion of the satellite, several coordinate systems are defined. As depicted in Fig. 1 , the frame $\mathcal{I}$ is attached to the camera. Reference frames are denoted by $\mathcal{F}_{j}^{*}$ $(j=1,2, \ldots, \infty)$, which are associated with the planes $\pi_{j}^{*}$ that are attached to the different faces of the satellite. The frames are numbered in ascending order based on the order that they enter the FOV. The feature points have coordinates $s_{j i} \in \mathbb{R}^{3}(i=1,2,3,4)$ in the $\mathcal{F}_{j}^{*}$ coordinate frame. The vector from the origin of $\mathcal{I}$ to the origin of $\mathcal{F}_{j}^{*}$ is $x_{f j}^{*} \in \mathbb{R}^{3}$, where $x_{f j}^{*}$ is measured in the $\mathcal{I}$ coordinate frame, and the rotation from $\mathcal{F}_{j}^{*}$ to $\mathcal{I}$ is $R_{j}^{*} \in \mathrm{SO}(3)$. The geometry between the coordinate frames can be used to determine the initial Euclidean positions of the $j$ th frame's feature points:

$$
\bar{m}_{j i}^{*}=x_{f j}^{*}+R_{j}^{*} s_{j i}
$$

where $\bar{m}_{j i}^{*} \in \mathbb{R}^{3}$ denotes the Euclidean coordinates of the feature points on the plane $\pi_{j}^{*}$ expressed in $\mathcal{I}$ :

$$
\bar{m}_{j i}^{*} \triangleq\left[\begin{array}{lll}
x_{j i}^{*} & y_{j i}^{*} & z_{j i}^{*}
\end{array}\right]^{T}
$$

The planes and coordinate frames that include an asterisk in the notation denote static instances of a moving plane or coordinate frame. Specifically, the asterisk is used to denote the static instant in time that the feature points that define a plane are first visible. As the plane or coordinate frame moves, the asterisk is dropped, to denote the moving plane/frame. For example, as $\pi_{j}^{*}$ moves from its reference position when the feature points that define the plane are first visible, it is denoted by $\pi_{j}(t)$ and the frame attached to $\pi_{j}(t)$ is defined as $\mathcal{F}_{j}(t)$. The vector from the origin of $\mathcal{I}$ to the origin of $\mathcal{F}_{j}(t)$ and its rotation from $\mathcal{F}_{j}(t)$ to $\mathcal{I}$ are denoted by $x_{f j}(t) \in \mathbb{R}^{3}$ and $R_{j}(t) \in \mathrm{SO}(3)$, respectively. The Euclidean coordinates of the $j$ th frame's feature points after any rotation can be defined as

$$
\bar{m}_{j i}=x_{f j}+R_{j} s_{j i}
$$

where $\bar{m}_{j i}(t) \in \mathbb{R}^{3}$ denotes the Euclidean coordinates of the feature points of $\pi_{j}$ expressed in $\mathcal{I}$ :

$$
\bar{m}_{j i} \triangleq\left[\begin{array}{lll}
x_{j i} & y_{j i} & z_{j i}
\end{array}\right]^{T}
$$

After some algebraic manipulation of Eqs. (1) and (3), the expression for $\bar{m}_{j i}$ in Eq. (3) can be rewritten as

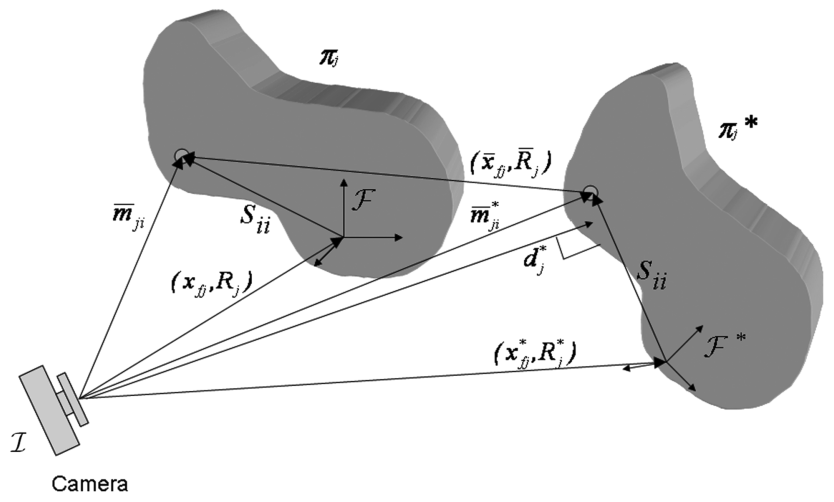

Fig. 1 Relationship between a coordinate frame in its initial position and all subsequent positions.

$$
\bar{m}_{j i}=\bar{x}_{f j}+\bar{R}_{j} \bar{m}_{j i}^{*}
$$

where $\bar{R}_{j}(t) \in \mathrm{SO}(3)$ and $\bar{x}_{f j}(t) \in \mathbb{R}^{3}$ are new rotation and translation variables, respectively, defined as

$$
\begin{gathered}
\bar{R}_{j}=R_{j} R_{j}^{* T} \\
\bar{x}_{f j}=x_{f j}-\bar{R}_{j} x_{f j}^{*}
\end{gathered}
$$

By using the projective relationship depicted in Fig. 1,

$$
d_{j}^{*}=n_{j}^{* T} \bar{m}_{j i}^{*}
$$

the expression in Eq. (ㄷ) can be expressed as

$$
\bar{m}_{j i}=\left(\bar{R}_{j}+\frac{\bar{x}_{f j}}{d_{j}^{*}} n_{j}^{* T}\right) \bar{m}_{j i}^{*}
$$

In Eq. (9), $n_{j}^{*} \in \mathbb{R}^{3}$ denotes the constant unit normal to the plane $\pi_{j}^{*}$ expressed in $\mathcal{I}$, and $d_{j}^{*}>0 \in \mathbb{R}$ is the distance to the plane along $n_{j}^{*}$.

\section{Euclidean Reconstruction}

\section{A. Homography Decomposition}

The relationship in Eq. (9) provides a means to quantify the translation and rotation between a coordinate system's location at two points in time and space. Because the position and orientation (i.e., pose) of $\mathcal{F}_{j}$ and $\mathcal{F}_{j}^{*}$ cannot be directly measured, a Euclidean reconstruction is developed in this section to obtain the position and rotation information by comparing multiple images acquired from the camera. Specifically, comparisons are made between $m_{j i}(t)$ and $m_{j i}^{*}$ in terms of $\mathcal{I}$. To facilitate the subsequent development, the normalized Euclidean coordinates of the feature points for $\mathcal{F}_{j}$ and $\mathcal{F}_{j}^{*}$ can be expressed in terms of $\mathcal{I}$ as $m_{j i}(t)$ and $m_{j i}^{*} \in \mathbb{R}^{3}$, respectively:

$$
m_{j i} \triangleq \frac{\bar{m}_{j i}}{z_{j i}} \quad m_{j i}^{*} \triangleq \frac{\bar{m}_{j i}^{*}}{z_{j i}^{*}}
$$

From Eqs. (9) and (10), the rotation and translation between the coordinate systems $\overline{\mathcal{F}}_{j}$ and $\mathcal{F}_{j}^{*}$ can be related in terms of the normalized Euclidean coordinates:

$$
m_{j i}=\underbrace{\frac{z_{j i}^{*}}{z_{j i}}}_{\alpha_{j i}} \underbrace{\left(\bar{R}_{j}+\frac{\bar{x}_{f j}}{d_{j}^{*}} n_{j}^{* T}\right)}_{H_{j}} m_{j i}^{*}
$$

In Eq. $(\underline{11}), \alpha_{j i}(t) \in \mathbb{R}$ denotes depth ratios, and $H_{j}(t) \in \mathbb{R}^{3 \times 3}$ denotes Euclidean homographies [27]. Each Euclidean feature point has a projected pixel coordinate expressed as

$$
p_{j i} \triangleq\left[\begin{array}{lll}
u_{j i} & v_{j i} & 1
\end{array}\right]^{T} \quad p_{j i}^{*} \triangleq\left[\begin{array}{lll}
u_{j i}^{*} & v_{j i}^{*} & 1
\end{array}\right]^{T}
$$


where $p_{j i}(t)$ represents the image-space coordinates of the timevarying feature points of $\mathcal{F}_{j}$, and $p_{j i}^{*} \in \mathbb{R}^{3}$ represents the imagespace coordinates of the feature points of $\mathcal{F}_{j}^{*}$. To calculate the Euclidean homography given in Eq. (11) from pixel information, the projected pixel coordinates are related to $m_{j i}(t)$ and $m_{j i}^{*}$ by the pinhole-camera model:

$$
p_{j i}=A m_{j i} \quad p_{j i}^{*}=A m_{j i}^{*}
$$

where $A \in \mathbb{R}^{3 \times 3}$ is a known, constant, invertible, intrinsic cameracalibration matrix. By using Eqs. (11) and (13), the following relationship can be developed:

$$
p_{j i}=\alpha_{j i}(\underbrace{\left(A H_{j} A^{-1}\right)}_{G_{j}} p_{j i}^{*}
$$

where $G_{j}(t) \in \mathbb{R}^{3 \times 3}$ denotes a projective homography. From four points, sets of linear equations can be developed from Eq. (14) to determine the projective homographies up to a scalar multiple. Techniques described in [7,28] can be used to decompose the Euclidean homographies to obtain $\alpha_{j i}(t), \bar{x}_{f j}(t) / d_{j}^{*}, \bar{R}_{j}(t)$, and $n_{j}^{* T}$ for $i=1,2,3,4$ and $j=1,2, \ldots, \infty$.

\section{B. Euclidean Reconstruction of the $\pi_{1}^{*}$ Plane}

Because of the fact that a distance is assumed to be known on the plane $\pi_{1}^{*}$, its Euclidean reconstruction is analyzed separately from the other planes. For the Euclidean reconstruction of subsequent planes that do not have an assumed known length, development is presented in Sec. III.C. To solve for the Euclidean coordinates $\bar{m}_{11}^{*}$ and $\bar{m}_{12}^{*}$, a new plane $\pi_{1}^{* \prime}$ is created parallel to $\pi_{1}^{*}$ containing the point $m_{11}^{*}$. This new plane will intersect a line $l_{1}^{*}$, which goes from the origin of $\mathcal{I}$ through $m_{12}^{*}$ and $\bar{m}_{12}^{*}$. The plane $\pi_{1}^{*^{\prime}}$ intersects $l_{1}^{*}$ at a point $m_{12}^{*^{\prime}} \in \mathbb{R}^{3}$. As depicted in Fig. 2, the distance between $m_{11}^{*}$ and $m_{12}^{* \prime}$ is defined as $\sigma_{1} \in \mathbb{R}$. The line $l_{1}^{*}$ and the plane $\pi_{1}^{* \prime}$ are defined by the sets of all points $\xi_{1}$ and $\xi_{2} \in \mathbb{R}^{3}$ that satisfy the implicit functions

$$
\begin{gathered}
\pi_{1}^{*^{\prime}}=\left\{\xi_{1} \mid-n_{1}^{*} \cdot\left(\xi_{1}-m_{11}^{*}\right)=0\right\} \\
l_{1}^{*}=\left\{\xi_{2} \mid \xi_{2}-u m_{12}^{*}=0\right\}
\end{gathered}
$$

where $u \in \mathbb{R}$ is some positive constant. The geometric relationships in Eqs. (15) and (16) indicate that any vector from one of the points $\xi_{1}$ on $\pi_{1}^{*^{\prime}}$ to $m_{11}^{*}$ is normal to $-n_{1}^{*}$, and that $l_{1}^{*}$ is the set of points $\xi_{2}$ that are linearly proportional to $m_{12}^{*}$ by some scalar $u \in \mathbb{R}$. From Eqs. (15) and (16), the point at which the $l_{1}^{*}$ and $\pi_{1}^{* \prime}$ intersect (i.e., when $\left.\xi_{1}=\overline{\xi_{2}}=m_{12}^{* \prime}\right)$ can be determined by solving Eq. (16) for $\xi_{2}$, substituting $\xi_{2}$ into Eq. (15) for $\xi_{1}$, solving the resulting expression for $u$, and then substituting the expression for $u$ into Eq. (16):

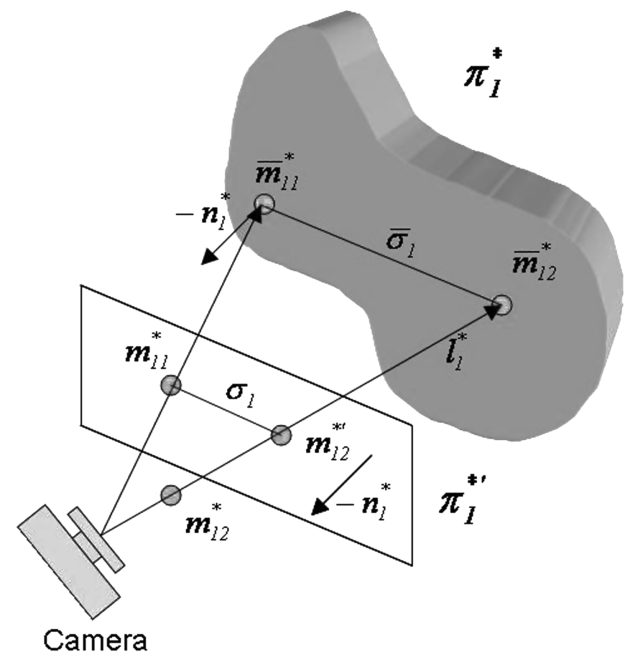

Fig. 2 Geometry used to extract the Euclidean position and orientation of feature points on a plane using a known distance.

$$
m_{12}^{* \prime}=\frac{-n_{1}^{*} \cdot m_{11}^{*}}{-n_{1}^{*} \cdot m_{12}^{*}} m_{12}^{*}
$$

The result in Eq. (17) can be used to solve for $\sigma_{1}$ :

$$
\sigma_{1}=\left\|m_{12}^{*^{\prime}}-m_{11}^{*}\right\|
$$

Because $\bar{\sigma}_{1} \in \mathbb{R}$ is assumed to be a known Euclidean distance between $\bar{m}_{11}^{*}$ and $\bar{m}_{12}^{*}$ and $\sigma_{1},\left\|m_{11}^{*}\right\|$ and $\left\|m_{12}^{*^{\prime}}\right\|$ can be determined as previously described, the similar triangle relationships derived from Fig. 2:

$$
\frac{\sigma_{1}}{\bar{\sigma}_{1}}=\frac{\left\|m_{11}^{*}\right\|}{\left\|\bar{m}_{11}^{*}\right\|}=\frac{\left\|m_{12}^{*^{\prime}}\right\|}{\left\|\bar{m}_{12}^{*}\right\|}
$$

can be used to recover $\bar{m}_{11}^{*}$ and $\bar{m}_{12}^{*}$ :

$$
\bar{m}_{11}^{*}=\frac{\left\|\bar{m}_{11}^{*}\right\|}{\left\|m_{11}^{*}\right\|} m_{11}^{*} \quad \bar{m}_{12}^{*}=\frac{\left\|\bar{m}_{12}^{*}\right\|}{\left\|m_{12}^{* \prime}\right\|} m_{12}^{* \prime}
$$

By using Eq. (20), the unknown constant scalar $d_{1}^{*}$ defined in Eq. (8) can be determined. Because $d_{1}^{*}$ is now known and $m_{1 i}^{*}$ is measurable, Eqs. ( $\underline{8})$ and (10) can be used to determine $z_{1 i}^{*}$ :

$$
z_{1 i}^{*}=\frac{d_{1}^{*}}{n_{1}^{* T} m_{1 i}^{*}}
$$

The Euclidean coordinates of the feature points on $\pi_{1}^{*}$ expressed in $\mathcal{I}$ can now be reconstructed by substituting Eq. (21) into Eq. (10):

$$
\bar{m}_{1 i}^{*}=\frac{d_{1}^{*}}{n_{1}^{* T} m_{1 i}^{*}} m_{1 i}^{*}
$$

To facilitate the subsequent daisy-chaining method, the translation from $\mathcal{I}$ to $\mathcal{F}_{1}^{*}$ and the orientation of $\mathcal{F}_{1}^{*}$ with respect to $\mathcal{I}$ can also be determined. Without loss of generality, the origin of $\mathcal{F}_{1}^{*}$ is assumed to be $\bar{m}_{11}^{*}$. The orientation of $\mathcal{F}_{1}^{*}$ with respect to $\mathcal{I}$ introduced in Eq. (1) can be expressed as

$$
R_{1}^{*}=\left[\begin{array}{lll}
i_{x 1} & i_{y 1} & i_{z 1}
\end{array}\right]
$$

In Eq. (23), $i_{x 1} \in \mathbb{R}^{3}$ and $i_{z 1} \in \mathbb{R}^{3}$ are defined as

$$
\begin{gathered}
i_{x 1}=\frac{\bar{m}_{12}^{*}-\bar{m}_{11}^{*}}{\bar{\sigma}_{1}} \\
i_{z 1}=-n_{1}^{*}
\end{gathered}
$$

and $i_{y 1} \in \mathbb{R}^{3}$ is defined as the cross product of $i_{x 1}$ and $i_{z 1}$ :

$$
i_{y 1}=-n_{1}^{*} \times \frac{\bar{m}_{12}^{*}-\bar{m}_{11}^{*}}{\bar{\sigma}_{1}}
$$

In Eqs. (25) and (26), the normal vector $n_{1}^{*}$ is known from the homography decomposition of Eqs. (11) and (14). Because $i_{z 1}$ is known from the homography decomposition of Eqs. (11) and (14), the results in Eq. (20) can be substituted into Eqs. (24) and (26) to determine $i_{x 1}$ and $i_{y 1}$. Based on Eq. (23), the rotation $\bar{R}_{1}^{*}$ can now be determined. Based on the fact that $R_{1}^{*}$ can be determined and $\bar{R}_{1}(t)$ is known from the homography decomposition of Eqs. (11) and (14), $R_{1}(t)$ can now be determined from Eq. (6). Because the origin of $\overline{\mathcal{F}}_{1}^{*}$ is selected as $\bar{m}_{11}^{*}$, the translation from $\overline{\mathcal{I}}$ to $\mathcal{F}_{1}^{*}$ introduced in Eq. (1) as $x_{f 1}^{*}$ is equal to $\bar{m}_{11}^{*}$, by definition. Given that $d_{1}^{*}, \bar{R}_{1}(t), \bar{x}_{f 1}(t) / \bar{d}_{1}^{*}$, and $x_{f 1}^{*}$ have been determined, Eq. (7) can be used to solve for $x_{f 1}(t)$.

\section{Euclidean Reconstruction of the $\pi_{j}^{*}$ Plane}

To exploit the daisy-chaining strategy to link the pose of $\mathcal{F}_{1}$ with the pose of the subsequent frames $\mathcal{F}_{j}(j=2,3, \ldots, \infty)$, the pose of $\mathcal{F}_{j}$ also needs to be determined. However, a known geometric length is not available on $\pi_{j}$, and so a method different from that in Sec. III.B must be developed. Specifically, in lieu of a known geometric length between feature points, the development in this section uses the 
previous reconstructed information to determine a geometric distance to the object that can be used to further support the Euclidean reconstruction of the subsequent feature points. Specifically, under the assumption that at some instant in time the $\pi_{j-1}$ plane and $\pi_{j}$ plane are both visible, the pose of $\mathcal{F}_{j-1}$ can be used to solve for the pose of $\mathcal{F}_{j}^{*}$, provided that the planes undergo some motion while they are both in the FOV. Let $t^{-} \in \mathbb{R}$ denote some instant in time when the two adjacent feature-point planes are both visible, and let $t^{+} \in \mathbb{R}$ denote some time after some incremental motion has occurred. Because $\mathcal{F}_{j-1}$ and $\mathcal{F}_{j}$ are part of the same rigid body, the rotation and translations for each of the planes are equal. Therefore, the known rotation

$$
R_{j-1}\left(t^{+}\right) R_{j-1}^{T}\left(t^{-}\right)
$$

is equal to $\bar{R}_{j}\left(t^{+}\right)$. Similarly, the known translation

$$
\bar{x}_{f(j-1)}\left(t^{+}\right)-\bar{x}_{f(j-1)}\left(t^{-}\right)
$$

is equal to $\bar{x}_{f j}\left(t^{+}\right)$. The facts that $\bar{x}_{f j}\left(t^{+}\right)$is known and the homography decomposition in Eq. (11) yields $\bar{x}_{f j}\left(t^{+}\right) / d_{j}^{*}$ can be used to determine $d_{j}^{*}$, which is the magnitude of the vector $\lambda_{j} \in \mathbb{R}^{3}$ that is defined from the origin of $\mathcal{I}$ to the plane $\pi_{j}^{*}$ along the normal $n_{j}^{*}$ (see Fig. 3).

Without loss of generality, the origin of the $\mathcal{F}_{j}^{*}$ frame is assigned to be $\bar{m}_{j 1}^{*}$. As indicated in Fig. $\underline{3}$, the angle between $\bar{m}_{j i}^{*}$ and $\lambda_{j}$ is defined to be $\theta_{j i} \in \mathbb{S}_{1}(i=1,2,3,4)$, which can be calculated using the definition of the dot product,

$$
\theta_{j i}=\cos ^{-1}\left(\frac{m_{j i}^{*} \cdot n_{j}^{*}}{\left\|m_{j i}^{*}\right\|\left\|n_{j}^{*}\right\|}\right)
$$

and hence the magnitude of $\bar{m}_{j i}^{*}$ can be determined as

$$
\left\|\bar{m}_{j i}^{*}\right\|=\frac{d_{j}^{*}}{\cos \theta_{j i}}
$$

Based on Eq. (28), $\bar{m}_{j i}^{*}$ can now be calculated as

$$
\bar{m}_{j i}^{*}=\frac{\left\|\bar{m}_{j i}^{*}\right\|}{\left\|m_{j i}^{*}\right\|} m_{j i}^{*}
$$

To facilitate the subsequent daisy-chaining method, the translation from $\mathcal{I}$ to $\mathcal{F}_{j}^{*}$ and the orientation of $\mathcal{F}_{j}^{*}$ with respect to $\mathcal{I}$ must be determined. As the satellite rotates, the $\pi_{1}$ plane will leave the FOV and a new plane will become visible. The orientation of $F_{j}^{*}$ with respect to $\mathcal{I}$ introduced in Eq. (3) can be expressed as

$$
R_{j}^{*}=\left[\begin{array}{lll}
i_{x j} & i_{y j} & i_{z j}
\end{array}\right]
$$

In Eq. (30), $i_{x j} \in \mathbb{R}^{3}$ and $i_{z j} \in \mathbb{R}^{3}$ are defined as

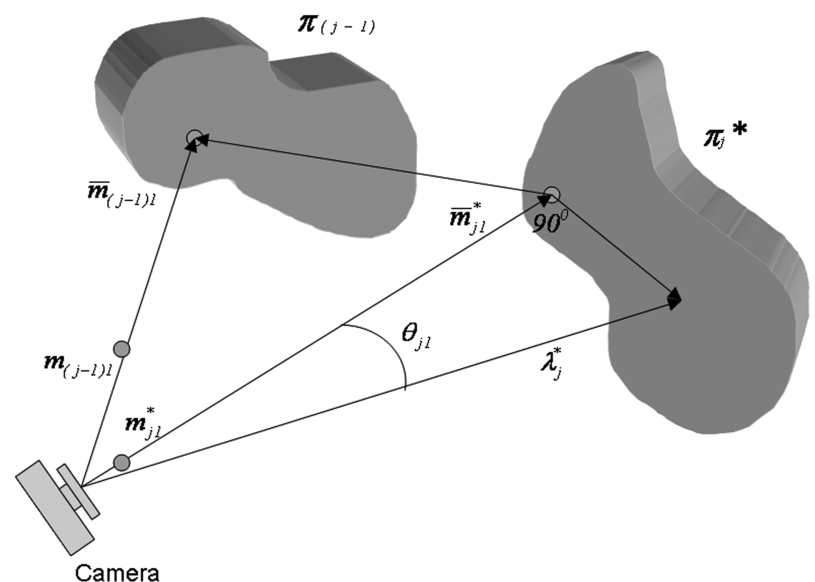

Fig. 3 Geometry used to extract the Euclidean position and orientation of a plane using a known plane.

$$
\begin{gathered}
i_{x j}=\frac{\bar{m}_{j 2}^{*}-\bar{m}_{j 1}^{*}}{\left\|\bar{m}_{j 2}^{*}-\bar{m}_{j 1}^{*}\right\|} \\
i_{z j}=-n_{j}^{*}
\end{gathered}
$$

and $i_{y j} \in \mathbb{R}^{3}$ is defined as the cross product of $i_{x j}$ and $i_{z j}$ :

$$
i_{y j}=-n_{j}^{*} \times \frac{\bar{m}_{j 2}^{*}-\bar{m}_{j 1}^{*}}{\left\|\bar{m}_{j 2}^{*}-\bar{m}_{j 1}^{*}\right\|}
$$

In Eqs. (32) and (33), the normal vector $n_{j}^{*}$ is known from the homography decomposition of Eqs. (11) and (14). Because $i_{z j}$ is known from the homography decomposition, the result in Eq. (29) can be substituted into Eqs. $\left(\underline{31)}\right.$ and (33) to determine $i_{x j}$ and $\bar{i}_{y j}$. Based on Eq. (30), the rotation $R_{j}^{*}$ can now be determined. Based on the fact that $R_{j}^{*}$ can be determined and $\bar{R}_{j}(t)$ is known from the homography decomposition of Eqs. (11) and (14), $R_{j}(t)$ can be determined. Because the origin of $\overline{\mathcal{F}_{j}^{*}}$ is selected as $\bar{m}_{j 1}^{*}$, the translation from $\mathcal{I}$ to $\mathcal{F}_{j}^{*}$, introduced in Eq. (3) as $x_{f j}^{*}$, is equal to $\bar{m}_{j 1}^{*}$, by definition. The fact that $d_{j}^{*}, \bar{R}_{j}(t), \bar{x}_{f j}(t) / d_{j}^{*}$ and $x_{f j}^{*}$ have been determined can now be used to solve for $x_{f j}(t)$.

\section{Daisy-Chaining}

The previous development can be used to determine the pose of $\mathcal{F}_{j}$ with respect to $\mathcal{I}$ when the feature points on $\pi_{j}$ are visible. The daisychaining method presented in this section is used to determine the relative pose between $\mathcal{F}_{1}$ and $\mathcal{F}_{j}$ so that the pose of $\mathcal{F}_{1}$ can still be determined even after the feature points on $\pi_{1}$ leave the FOV, provided that the feature points on $\pi_{j}$ are visible.

The relative pose between $\mathcal{F}_{j-1}$ and $\mathcal{F}_{j}$ will not change, because both time-varying coordinate frames are attached to the same rigid body. Hence, the relative pose between $\mathcal{F}_{j-1}$ and $\mathcal{F}_{j}$ only needs to be determined once. As in the previous sections, the subsequent development is based on the assumption that the feature points on both $\pi_{j-1}$ and $\pi_{j}$ are visible at some moment in time. The first instant when the feature points on both $\pi_{j-1}$ and $\pi_{j}$ are visible is when $\pi_{j}$ first enters the FOV, which is denoted as $\pi_{j}^{*}$. At this instant in time, the pose of $\mathcal{F}_{j}$ is denoted by $\mathcal{F}_{j}^{*}$, with a translation and rotation with respect to $\mathcal{I}$ denoted as $x_{f j}^{*}$ and $R_{j}^{*}$. At some instant when the feature points on $\pi_{1}$ are visible, a reference image is acquired that contains the geometric length $\bar{\sigma}_{1}$. Based on the methods in Sec. III.B, the Euclidean coordinates of the feature points on $\pi_{1}^{*} \overline{\text { can }}$ be reconstructed and expressed in $\mathcal{I}$. The subsequent images of the feature points on $\pi_{1}$ can then be used to construct the homography relationship given in Eq. (11), which allows the Euclidean coordinates of feature points on $\bar{\pi}_{1}$ to continue to be reconstructed. At some other point in time, the feature points on $\pi_{1}$ and $\pi_{2}^{*}$ are both in the FOV, and a second reference image is acquired. Assuming that the next image is acquired when both $\pi_{1}$ and $\pi_{2}^{*}$ have undergone some motion and the feature points on both planes are still visible, the methods in Sec. III.C can be used to reconstruct the Euclidean coordinates of the feature points on $\pi_{2}^{*}$ expressed in $\mathcal{I}$. The homography relationship given in Eq. (11) can then be used to determine the relative pose of $\mathcal{F}_{2}$ with respect to $\mathcal{F}_{2}^{*}$ expressed in $\mathcal{I}$, as well as the pose of $\mathcal{F}_{2}$ with respect to $\mathcal{I}$ expressed in $\mathcal{I}$. Given the Euclidean coordinates of the feature points on $\pi_{1}$ and $\pi_{2}^{*}$ expressed in $\mathcal{I}$, the constant relative pose of $\mathcal{F}_{1}$ with respect to $\mathcal{F}_{2}$ can be determined. That is, the constant vector $x_{21}^{\mathcal{F}_{2}} \in \mathbb{R}^{3}$ from the origin of $\mathcal{F}_{2}$ to the origin of $\mathcal{F}_{1}$ expressed in $\mathcal{F}_{2}$ is determined when $\mathcal{F}_{2}$ and $\mathcal{F}_{2}^{*}$ are coincident:

$$
x_{21}^{\mathcal{F}_{2}}=R_{2}^{* T}\left(x_{f 1}-x_{f 2}^{*}\right)
$$

In Eq. (34), $R_{2}^{* T}$ is used to express the difference $x_{f 1}-x_{f 2}^{*}$ in $\mathcal{F}_{2}$. The vector $\overline{x_{21}}$ is constant in $\mathcal{F}_{2}$, but is time-varying when expressed in $\mathcal{I}$. The constant rotation $R_{\mathcal{F} 1}^{\mathcal{F} 2} \in \mathrm{SO}(3)$ from $\mathcal{F}_{1}$ to $\mathcal{F}_{2}$ is determined when $\mathcal{F}_{2}$ and $\mathcal{F}_{2}^{*}$ are coincident: 


$$
R_{\mathcal{F} 1}^{\mathcal{F} 2}=R_{2}^{* T} R_{1}
$$

As the satellite continues to rotate, the feature points in $\pi_{1}$ will leave the FOV, and the feature points on $\pi_{2}$ and $\pi_{3}^{*}$ will eventually be in the FOV at the same time. A third reference image is now acquired. To estimate the pose of $\mathcal{F}_{1}$ and the Euclidean feature points of $\pi_{1}$, the vector $x_{f 1}$ from the origin of $\mathcal{I}$ to the origin of $\mathcal{F}_{1}$, as well as the $R_{1}$ rotation from $\mathcal{F}_{1}$ to $\mathcal{I}$, are required. As stated earlier, the pose of $\mathcal{F}_{2}$ with respect to $\mathcal{I}$ expressed in $\mathcal{I}$ has been determined, and so the vector from the origin of $\mathcal{F}_{2}$ to the origin of $\mathcal{F}_{1}$ can be expressed in $\mathcal{I}$ as

$$
x_{21}^{\mathcal{I}}=R_{2} x_{21}^{\mathcal{F}_{2}}
$$

even though $\pi_{1}$ is no longer in the FOV. The vector from the origin of $\mathcal{I}$ to the origin of $\mathcal{F}_{1}$ is equivalent to the vector from the origin of $\mathcal{I}$ to the origin of $\mathcal{F}_{2}$ plus the vector from the origin of $\mathcal{F}_{2}$ to the origin of $\mathcal{F}_{1}$. Both of these vectors are known, and so the vector from the origin of $\mathcal{I}$ to the origin of $\mathcal{F}_{1}$ can be determined as

$$
x_{f 1}=x_{f 2}+x_{21}^{\mathcal{I}}
$$

The rotation from $\mathcal{F}_{1}$ to $\mathcal{I}$ is equivalent to the rotation from $\mathcal{F}_{2}$ to $\mathcal{I}$ multiplied by the rotation from $\mathcal{F}_{1}$ to $\mathcal{F}_{2}$. Both of these rotations are known, and so the rotation from $\mathcal{F}_{1}$ to $\mathcal{I}$ can be determined as

$$
R_{1}=R_{2} R_{\mathcal{F} 1}^{\mathcal{F} 2}
$$

Assuming that the next image is acquired when both $\pi_{2}$ and $\pi_{3}^{*}$ have undergone some motion and the feature points on both planes are still visible, then the methods in Sec. III.C can be used to reconstruct the Euclidean coordinates of the feature points on $\pi_{3}^{*}$ expressed in $\mathcal{I}$. The homography relationship given in Eq. (11) can then be used to determine the relative pose of $\mathcal{F}_{3}$ with respect to $\mathcal{F}_{3}^{*}$ expressed in $\mathcal{I}$, as well as the pose of $\mathcal{F}_{3}$ with respect to $\mathcal{I}$ expressed in $\mathcal{I}$. Given the Euclidean coordinates of the feature points on $\pi_{2}$ and $\pi_{3}^{*}$ expressed in $\mathcal{I}$, the constant relative pose of $\mathcal{F}_{2}$ with respect to $\mathcal{F}_{3}$ can be determined; that is, the constant vector $x_{32}^{\mathcal{F}_{3}} \in \mathbb{R}^{3}$ from the origin of $\mathcal{F}_{3}$ to the origin of $\mathcal{F}_{2}$ expressed in $\mathcal{F}_{3}$ is determined when $\mathcal{F}_{3}$ and $\mathcal{F}_{3}^{*}$ are coincident:

$$
x_{32}^{\mathcal{F}_{3}}=R_{3}^{* T}\left(x_{f 2}-x_{f 3}^{*}\right)
$$

The constant rotation $R_{\mathcal{F} 2}^{\mathcal{F} 3} \in \mathrm{SO}(3)$ from $\mathcal{F}_{2}$ to $\mathcal{F}_{3}$ that is determined when $\mathcal{F}_{3}$ and $\mathcal{F}_{3}^{*}$ are coincident is

$$
R_{\mathcal{F} 2}^{\mathcal{F} 3}=R_{3}^{* T} R_{2}
$$

The constant relative pose of $\mathcal{F}_{1}$ with respect to $\mathcal{F}_{3}$ can also be determined. The constant vector $x_{31}^{\mathcal{F}_{3}} \in \mathbb{R}^{3}$ from the origin of $\mathcal{F}_{3}$ to the origin of $\mathcal{F}_{1}$ expressed in $\mathcal{F}_{3}$ is equivalent to the vector from the origin of $\mathcal{F}_{3}$ to the origin of $\mathcal{F}_{2}$ plus the vector from the origin of $\mathcal{F}_{2}$ to the origin of $\mathcal{F}_{1}$, both expressed in $\mathcal{F}_{3}$. Both of these vectors are known, and so the vector from the origin of $\mathcal{F}_{3}$ to the origin of $\mathcal{F}_{1}$ expressed in $\mathcal{F}_{3}$ can be determined as

$$
x_{31}^{\mathcal{F} 3}=x_{32}^{\mathcal{F} 3}+x_{21}^{\mathcal{F} 3}=R_{3}^{* T}\left(x_{f 2}-x_{f 3}^{*}\right)+R_{3}^{* T} R_{2} x_{21}^{\mathcal{F} 2}
$$

The constant rotation $R_{\mathcal{F} 1}^{\mathcal{F} 3} \in \mathrm{SO}(3)$ from $\mathcal{F}_{1}$ to $\mathcal{F}_{3}$ is equivalent to the rotation from $\mathcal{F}_{2}$ to $\mathcal{F}_{3}$ multiplied by the rotation from $\mathcal{F}_{1}$ to $\mathcal{F}_{2}$. Both of these rotations are known, and so the rotation from $\mathcal{F}_{1}$ to $\mathcal{F}_{3}$, determined when $\mathcal{F}_{3}$ and $\mathcal{F}_{3}^{*}$ are coincident, is

$$
R_{\mathcal{F} 1}^{\mathcal{F} 3}=R_{\mathcal{F} 2}^{\mathcal{F} 3} R_{\mathcal{F} 1}^{\mathcal{F} 2}
$$

This procedure can be continued indefinitely to calculate the vector from the origin of $\mathcal{I}$ to the origin of the $\mathcal{F}_{1}$ frame expressed in $\mathcal{I}$. The general equation for $x_{f 1}(t)$ after $j$ rotations is

$$
x_{f 1}=x_{f j}+R_{j} x_{j 1}^{\mathcal{F} j}
$$

Likewise, the general equation for $R_{1}(t)$ after $j$ rotations is

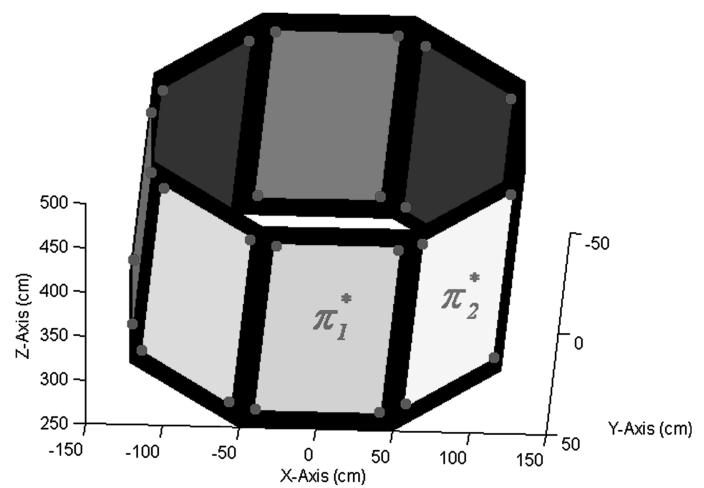

Fig. 4 Virtual satellite with feature points created in MATLAB.

$$
R_{1}=R_{j} R_{\mathcal{F} 1}^{\mathcal{F} j}
$$

The Euclidean coordinates of the feature points on $\pi_{1}$ after $j$ rotations can also be determined as

$$
\bar{m}_{1 i}=x_{f j}+R_{j} x_{j 1}^{\mathcal{F} j}+R_{j} R_{\mathcal{F} 1}^{\mathcal{F} j} s_{1 i}
$$

where $x_{j 1}^{\mathcal{F}_{j}}(t) \in \mathbb{R}^{3}$ is the vector from the origin of $\mathcal{F}_{j}(t)$ to the origin of $\mathcal{F}_{1}(t)$, and $R_{\mathcal{F}_{1}}^{\mathcal{F}_{j}} \in \mathrm{SO}(3)$ is the rotation from $\mathcal{F}_{j}(t)$ to $\mathcal{F}_{1}(t)$.

\section{Numerical Simulation}

\section{A. Simulation Setup}

The geometric and daisy-chaining method developed in Sec. IV was implemented in a MATLAB simulation. The uniform octagonal satellite depicted in Fig. 4 was created and rotated 51 times about its axis of symmetry by $0.4 \overline{3} 63 \mathrm{rad}(25 \mathrm{deg})$. Each of the square sides measures 1 by $1 \mathrm{~m}$. Smaller square sections are centered on each side and the feature points were chosen to be the corners of these sections. The symmetry of the object is coincidental and not required for the feature-point calculations. Also, planar and nonplanar surfaces can exist on the same object, but to form a homography matrix, four feature points on a planar surface or eight nonplanar points are needed. To simplify our analysis, four planar feature points are given without loss of generality. The $\pi_{1}^{*}$ plane rotated a total $30.1069 \mathrm{rad}$ $(1275 \mathrm{deg})$, left and reentered the FOV three times, and formed 29 image chains. The satellite was viewed by a camera with a scaled focal length of 860 pixels, and the pixel coordinates of the principle point were chosen to be $[360,240]$ pixels. Although the simulation was implemented for a rotating satellite, the developed method can be applied to a general rotating and translating rigid body.

\section{B. Results and Discussion}

The Euclidean errors of estimated feature points with no pixel noise and a perfect camera calibration are plotted in Fig. 5 versus the number of daisy chains between images. The errors were calculated by subtracting the estimated Euclidean feature-point location [Eq. (44)] from the actual Euclidean position for each axis. The actual Euclidean position was known for the simulation and was only used to quantify the estimation error. The errors grew as the chain number grew, but were all on the order of $10^{-6} \mathrm{~cm}$, resulting in a near-perfect estimate, and any discrepancies were due to numeric noise. The simulation was also executed assuming a nonperfect camera calibration. Random error was introduced into the calibration in the form of a uniform distribution on the interval $[-5,5]$ pixels. Motivation for this degree of calibration uncertainty was derived from the fact that typical calibration routines yield results well below the simulated uncertainty. For this camera-calibration routine, errors in the scaled focal length of \pm 1 pixel and errors in the principle point of \pm 2 pixels are not uncommon. The Euclidean estimation errors are

[Data available online at http://www.vision.caltech.edu/bouguetj/calib_doc/ [retrieved November 2007]. 

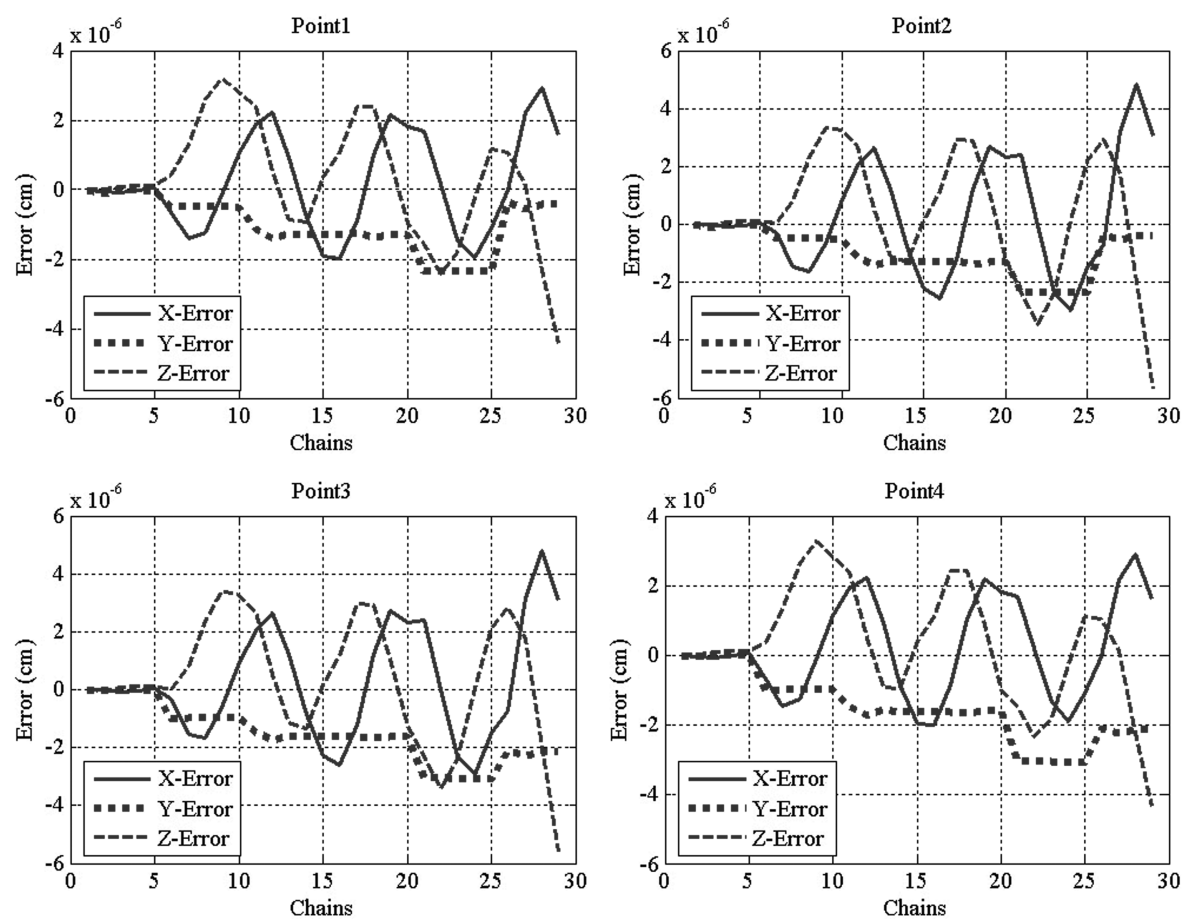

Fig. 5 Errors for each point versus chain number for a perfect camera calibration and no pixel noise.
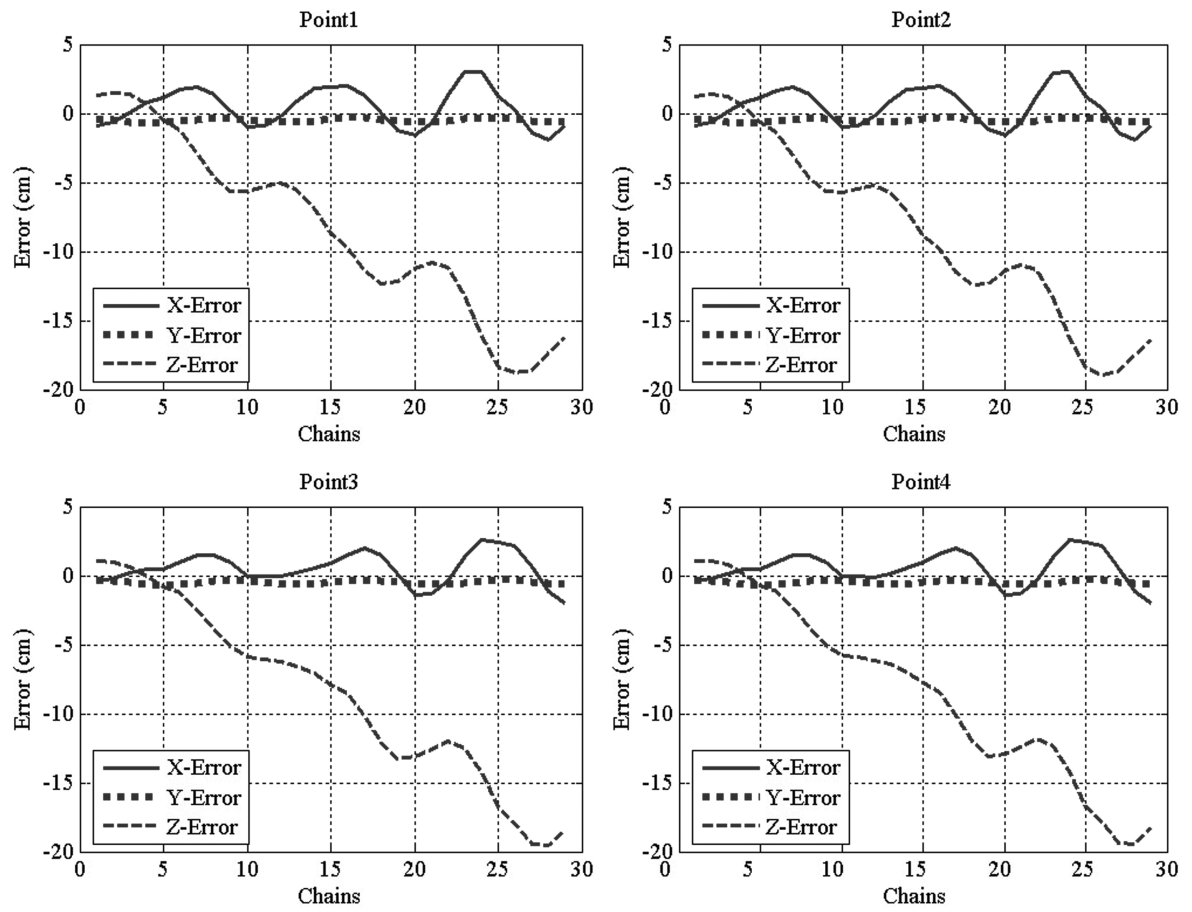

Fig. 6 Errors for each point versus chain number for a nonperfect camera calibration.

plotted versus the chain number in Fig. 6. As in Fig. 5, the errors grew as the chain number increased. This behavior is expected, due to the fact that in any estimation algorithm in which the feature points permanently leave the FOV, the error will continue to propagate. The errors along the $Y$ axis are less than the errors along the other axes, due to the rotation being about the $Y$ axis, which is the axis of symmetry. Future efforts will focus on mitigating the propagation of the estimation error along the $X$ and $Z$ axes. Some possible approaches include resetting the estimate when a previously viewed image returns to the FOV and linear filtering methods. To illustrate the possible utility of this future work, we simulated a simple ad hoc resetting approach for the uncertain camera-calibration case.
Whenever the first frame reenters the FOV, the measured value replaced the estimated value. In this ad hoc demonstration, our knowledge of the exact location of the satellite was used to determine when the satellite reentered the FOV. The Euclidean estimation errors are plotted versus the chain number in Fig. 7. As in Fig. 7 the errors grew as the chain number increased, but due to the estimates being reset (denoted by a circle in Fig. 7), they remain bounded.

\section{Conclusions}

A new daisy-chaining method is combined with new image geometry techniques to track the Euclidean position of the feature 

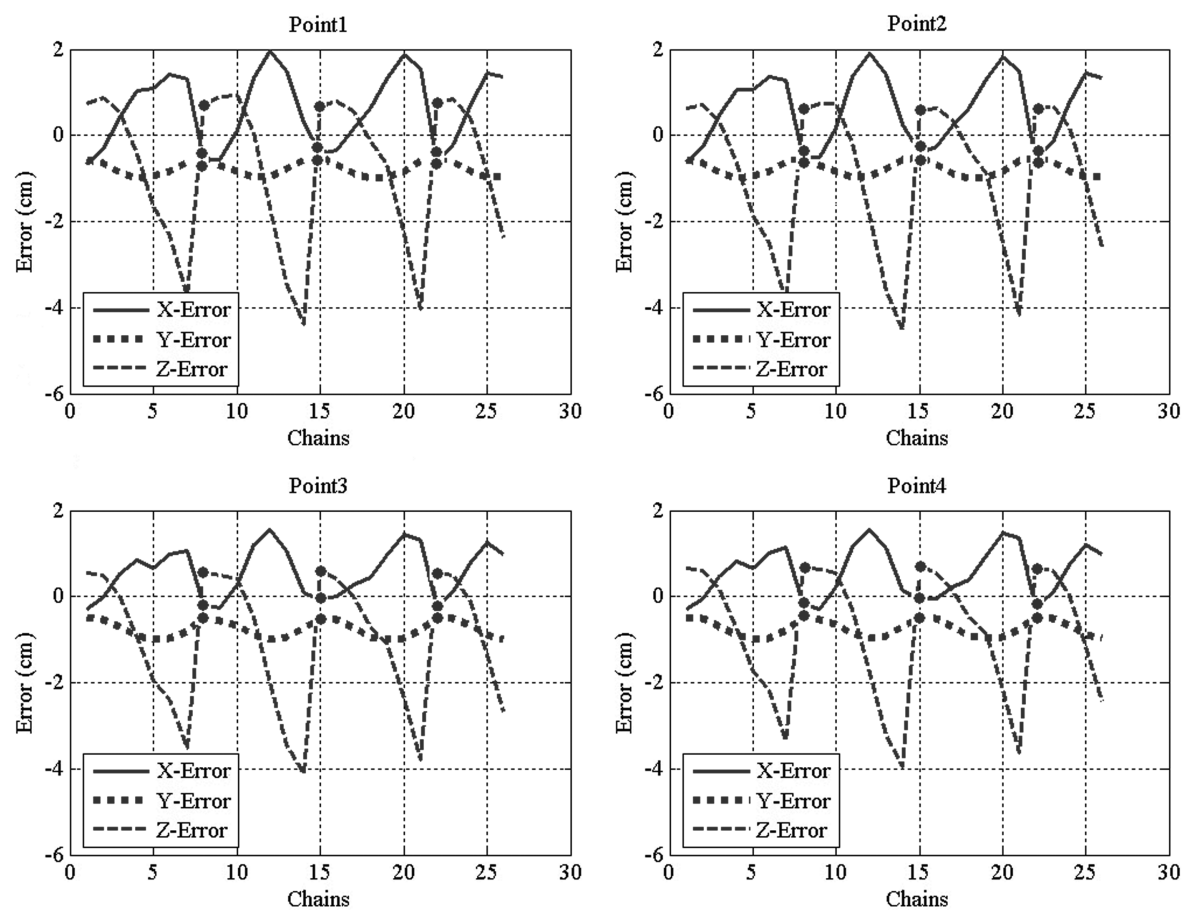

Fig. 7 Errors for each point versus chain number for a nonperfect camera calibration with error resetting.

points of a rotating and translating body despite the potential to leave the field of view or become occluded. These methods allow for relationships between frames to be determined as the rigid body moves. By chaining the developed relationships to the inertial camera frame, the Euclidean position of the feature points can be estimated. This method shows promise, but some limitations are apparent. For example, the numerical simulation demonstrates the effectiveness of the method, but the errors have the potential to accumulate (as in dead-reckoning navigation) if the feature points leave the field of view and never return. The algorithm is also susceptible to errors in the camera-calibration matrix. Future work is required to alleviate these errors.

\section{Acknowledgments}

This research is supported in part by the National Science Foundation's Career Award CMS-0547448, U.S. Air Force Office of Scientific Research contracts F49620-03-1-0381 and F49620-03-10170, U.S. Air Force Research Laboratory contract FA4819-05-D0011, and the U.S. Department of Energy's University Research Program in Robotics (URPR) grant DE-FG04-86NE37967.

\section{References}

[1] Chesi, G., Hashimoto, K., Prattichizzo, D., and Vicino, A., "Keeping Features in the Field of View in Eye-in-Hand Visual Servoing: A Switching Approach," IEEE Transactions on Robotics and Automation, Vol. 20, No. 5, 2004, pp. 908-914. doi:10.1109/TRO.2004.829456

[2] Gans, N. R., and Hutchinson, S. A., "A Switching Approach to Visual Servo Control," Proceedings of the 2002 IEEE International Symposium on Intelligent Control, Inst. of Electrical and Electronics Engineers, Piscataway, NJ, 2002, pp. 770-776.

[3] Chen, J., Dawson, D. M., Dixon, W. E., and Chitrakaran, V. K., "Navigation Function-Based Visual Servo Control," Automatica, Vol. 43, No. 7, 2007, pp. 1165-1177. doi:10.1016/j.automatica.2006.12.018

[4] Cowan, N., Weingarten, J., and Koditschek, D., "Visual Servoing Via Navigation Functions," IEEE Transactions on Robotics and Automation, Vol. 18, No. 4, 2002, pp. 521-533. doi:10.1109/TRA.2002.802202

[5] Mezouar, Y., and Chaumette, F., "Path Planning for Robust ImageBased Control," IEEE Transactions on Robotics and Automation, Vol. 18, No. 4, 2002, pp. 534-549. doi:10.1109/TRA.2002.802218
[6] Mezouar, Y., and Chaumette, F., "Optimal Camera Trajectory with Image-Based Control," The International Journal of Robotics Research, Vol. 22, No. 10, 2003, pp. 781-803. doi: $10.1177 / 027836490302210001$

[7] Zhang, Z., and Hanson, A., "Scaled Euclidean 3D Reconstruction Based on Externally Uncalibrated Cameras," Proceedings of the IEEE International Symposium on Computer Vision, Inst. of Electrical and Electronics Engineers, Piscataway, NJ, 1995, pp. 37-42.

[8] Corke, P. I., and Hutchinson, S. A., "A New Partitioned Approach to Image-Based Visual Servo Control," IEEE Transactions on Robotics and Automation, Vol. 17, No. 4, 2001, pp. 507-515. doi: $10.1109 / 70.954764$

[9] Benhimane, S., and Malis, E., "Vision-Based Control with Respect to Planar and Nonplanar Objects Using a Zooming Camera," Proceedings of the IEEE International Conference on Advanced Robotics, Inst. of Electrical and Electronics Engineers, Piscataway, NJ, 2003, pp. 991996.

[10] Hsu, S., Harpreet, H. S., and Kumar, R., "Automated Mosaics via Topology Inference," IEEE Computer Graphics and Applications, Vol. 22, No. 2, 2002, pp. 44-54. doi: $10.1109 / 38.988746$

[11] Schechner, Y., and Nayar, S., "Generalized Mosaicing: High Dynamic Range in a Wide Field of View," International Journal of Computer Vision, Vol. 53, No. 3, 2003, pp. 245-267. doi:10.1023/A:1023082924255

[12] Smolic, A., and Wiegand, T., "High-Resolution Video Mosaicing," Proceedings of the IEEE International Conference on Image Processing, Inst. of Electrical and Electronics Engineers, Piscataway, NJ, 2001, pp. 872-875.

[13] Irani, M., Anandan, P., Bergen, J., Kumar, R., and Hsu, S., "Efficient Representations of Video Sequences and their Applications," Signal Processing: Image Communication;Polymer-Plastics Technology and Engineering, Vol. 8, No. 4, May 1996, pp. 327-351. doi:10.1016/0923-5965(95)00055-0

[14] Swarninathan, R., and Nayar, S., "Non-Metric Calibration of WideAngle Lenses and Polycameras," IEEE Computer Society Conference on Computer Vision and Pattern Recognition, IEEE Computer Society, Los Alamitos, CA, 1999, pp. 413-419.

[15] Chiuso, A., Favaro, P., Jin, H., and Soatto, S., "Structure from Motion Causally Integrated Over Time," IEEE Transactions on Pattern Analysis and Machine Intelligence, Vol. 24, No. 4, 2002, pp. 523-535. doi:10.1109/34.993559

[16] Garcia-Aracil, N., Malis, E., Aracil-Santonja, R., and Perez-Vidal, C., "Continuous Visual Servoing Despite the Changes of Visibility in Image Features," IEEE Transactions on Robotics and Automation, Vol. 21, No. 6, 2005, pp. 1214-1220. doi: $10.1109 /$ TRO.2005.855995 
[17] Isard, M., and Blake, A., "Condensation-Conditional Density Propagation for Visual Tracking," International Journal of Computer Vision, Vol. 29, No. 1, 1998, pp. 5-28. doi:10.1023/A:1008078328650

[18] Camps, O., Lim, H., Mazzaro, C., and Sznaier, M., "A CaratheodoryFejer Approach to Robust Multiframe Tracking," Proceedings of the IEEE International Conference on Computer Vision, Inst. of Electrical and Electronics Engineers, Piscataway, NJ, 2003, pp. 1048-1055.

[19] Lublinerman, R., Sznaier, M., and Camps, O., "Dynamics Based Robust Motion Segmentation," IEEE Computer Society Conference on Computer Vision and Pattern Recognition, IEEE Computer Society, Los Alamitos, CA, 2006, pp. 1176-1184.

[20] Morariu, V., and Camps, O., "Modeling Correspondences for MultiCamera Tracking Using Nonlinear Manifold Learning and Target Dynamics," IEEE Computer Society Conference on Computer Vision and Pattern Recognition, IEEE Computer Society, Los Alamitos, CA, 2006, pp. 545-552.

[21] Niethammer, M., Tannenbaum, A., and Angenent, S., "Dynamic Active Contours for Visual Tracking," IEEE Transactions on Automatic Control, Vol. 51, No. 4, 2006, pp. 562-579. doi:10.1109/TAC.2006.872837

[22] North, B., Blake, A., Isard, M., and Rittscher, J., "Learning and Classification of Complex Dynamics," IEEE Transactions on Pattern Analysis and Machine Intelligence, Vol. 22, No. 9, 2000, pp. 10161034.

doi: $\underline{10.1109 / 34.877523}$
[23] Dupree, K., Gans, N. R., MacKunis, W., and Dixon, W. E., "Euclidean Calculation of Feature Points of a Rotating Satellite: A Daisy Chaining Approach," Proceedings of the American Control Conference, American Automatic Control Council, Evanston, IL, 2007, pp. 3874-3879.

[24] Hu, G., Mehta, S., Gans, N. R., and Dixon, W., "Daisy Chaining Based Visual Servo Control, Part 1: Adaptive Quaternion-Based Tracking Control," Proceedings of the 2007 IEEE International Conference on Control Applications, Inst. of Electrical and Electronics Engineers, Piscataway, NJ, 2007, pp. 1474-1479.

[25] Hu, G., Gans, N. R., Mehta, S., and Dixon, W. E., "Daisy Chaining Based Visual Servo Control, Part 2: Extensions, Applications and Open Problems," Proceedings of the 2007 IEEE International Conference on Control Applications, Inst. of Electrical and Electronics Engineers, Piscataway, NJ, 2007, pp. 729-734.

[26] Malis, E., and Chaumette, F., "2 1/2 D Visual Servoing with Respect to Unknown Objects Through a New Estimation Scheme of Camera Displacement," International Journal of Computer Vision, Vol. 37, No. 1, 2000, pp. 79-97. doi:10.1023/A:1008181530296

[27] Faugeras, O., Three-Dimensional Computer Vision, MIT Press, Cambridge, MA, 2001.

[28] Faugeras, O., and Lustman, F., "Motion and Structure From Motion in a Piecewise Planar Environment," International Journal of Pattern Recognition and Artificial Intelligence, Vol. 2, No. 3, 1988, pp. 485508 . doi: $10.1142 / \mathrm{S} 0218001488000285$ 